\title{
BEHRINGER, Wolfgang, Im Zeichen des Merkur. Reichspost und Kommunikationsrevolution in der Frühen Neuzeit
}

\section{Christophe Duhamelle}

\section{OpenEdition}

\section{Journals}

Édition électronique

URL : http://journals.openedition.org/ifha/1021

DOI : 10.4000/ifha.1021

ISSN : 2198-8943

\section{Éditeur}

IFRA - Institut franco-allemand (sciences historiques et sociales)

\section{Référence électronique}

Christophe Duhamelle, "BEHRINGER, Wolfgang, Im Zeichen des Merkur. Reichspost und Kommunikationsrevolution in der Frühen Neuzeit », Revue de l'IFHA [En ligne], Date de recension, mis en ligne le 01 janvier 2004, consulté le 22 septembre 2020. URL : http://journals.openedition.org/ifha/ 1021 ; DOI : https://doi.org/10.4000/ifha.1021

Ce document a été généré automatiquement le 22 septembre 2020.

(CIFHA 


\title{
BEHRINGER, Wolfgang, Im Zeichen des Merkur. Reichspost und Kommunikationsrevolution in der Frühen Neuzeit
}

\author{
Christophe Duhamelle
}

1 Cito, cito, cito! Vite, vite, vite ! L'invitation figurait déjà sur les premières feuilles de route, à pointer de poste en poste, des cavaliers portant le courrier autour de Milan, dans les années 1380. Elle demeura l'emblème de cette institution nouvelle, la poste, « matrice de tous les processus de communication standardisés » (p. 683), source d'une culture du temps et de l'espace fondée sur la vitesse, la régularité et le goût de la nouvelle. Le travail magistral de W.B. va bien au-delà d'une description institutionnelle de la poste impériale à l'époque moderne. Mêlant érudition et hauteur de vue, il démontre que le temps et l'espace à l'époque moderne ne racontent pas une histoire immobile. Il aborde successivement la mise en place du réseau postal, la « révolution des médias " puis celle des transports qui se greffent sur la poste, avant d'étudier les novations organisationnelles et de conclure " sous le signe de Mercure ", le Dieu ailé s'étant vite imposé comme le symbole du message transmis toujours plus vite.

2 Cette histoire est d'abord celle d'une institution d'Empire - une dimension que W.B. prend en compte, ce qui tranche avec un cloisonnement dommageable entre les spécialistes des institutions impériales et les autres. Créée vers 1490 comme service personnel de l'empereur et confiée à la famille italienne des Tassis, la poste a son siège principal à Bruxelles. Elle prend forme avec un traité de 1516 - mais celui-ci, centré sur les Pays-Bas, est plus habsbourgeois qu'impérial. La séparation entre les deux branches des Habsbourg et les guerres internes au Saint-Empire entraînent, au terme d'une évolution conflictuelle, la véritable création en 1597 par un mandat de Rodolphe II de la kaiserliche Reichspost (expression intraduisible, puisqu'il faudrait rendre en deux mots la dualité ici exprimée entre l'empereur et l'Empire, c'est-à-dire l'ensemble des territoires). Supraterritoriale et pluriconfessionnelle, elle n'est plus la chose des 
Habsbourg (l'Autriche est d'ailleurs la première à créer sa propre poste hors de ce système) ; le siège quitte les Pays-Bas pour Francfort au début du XVIIIe s., puis Ratisbonne en 1748. Le " généralat des postes " est transformé en fief héréditaire d'Empire en 1615 et les Tassis, désormais von Thurn und Taxis, achètent en 1772 un comté immédiat bientôt " princifié ». La poste, transcendant les états territoriaux, est ainsi en même temps un état territorial, et sa fonction de truchement s'exerce aussi au niveau politique, puisque les Thurn und Taxis, après 1748, sont " commissaires principaux " de l'empereur à la Diète d'Empire de Ratisbonne. La poste impériale n'échappe donc pas aux querelles pour le partage des pouvoirs entre Empire et princes :W.B. détaille les controverses sur la Postregal qui culminent en 1658-1662 et montre les deux pôles de résistance à la supraterritorialité de la poste. Il s'agit au départ des villes d'Empire, rétives (sauf Augsbourg) et enclines à organiser leurs propres systèmes de messagerie, si bien que la poste impériale eut longtemps pour caractéristique d'éviter les villes principales (Ulm ne devint ainsi un centre postal qu'en 1680). Il s'agit surtout des plus grandes principautés qui, après la paix de Westphalie, s'arrogent un monopole postal (Brandebourg en 1649, Saxe en 1651, Brunswick-Lunebourg et HesseKassel en 1652) et pour créer leurs propres réseaux congédient la poste impériale. Celle-ci doit se contenter du reste mais, devient, par là-même, un pôle de résistance des petits états d'Empire contre les gros ainsi qu'une des rares institutions impériales inscrites dans le quotidien et imposant, par ses aigles, ses décorations, ses uniformes, sa couleur jaune, « la présence optique de l'empereur et de l'Empire au XVIIIe s. »(p. 618).

3 Sa position institutionnelle complexe et contestée conféra à la poste une grande autonomie et la confronta à l'aiguillon de la concurrence, engendrant une densité " sans égale en Europe » (p. 279) du réseau postal. Cette histoire est donc aussi celle d'une inventivité constante, surtout après la transformation en fief héréditaire - cet " archaïsme " institutionnel étant paradoxalement la condition d'une modernisation accrue. W.B. retrace la mise en place d'un service standardisé et fiable : régularité des courriers, ouverture au public, tarification claire, stabilisation des trajets, puis passage au transport des personnes et établissement, au milieu du XVIIe s. (mais c'est un échec), puis après 1697 avec un succès croissant, d'un service de coches impériaux. Quelques grands commis ont marqué cette histoire, comme Johannes von den Birghden dans les tourmentes de la guerre de Trente Ans, ou Joseph Heger, introducteur du billet de transport, qui assure au milieu du XVIIIe s. la supériorité des voitures de la poste sur tous leurs concurrents. Soudé par une position juridique supraterritoriale, le personnel de la poste développe très tôt un esprit de corps (première grève générale en 1579) et une éthique professionnelle appuyée sur des procédures écrites et des inspections régulières ainsi que sur la recherche d'une efficacité croissante et le refus d'accorder un traitement spécial à quelque client que ce soit. Certaines familles y font une ascension sociale spectaculaire, mais restent au service de la poste, s'alliant et parfois se déchirant entre elles. La poste impériale ne cesse ainsi de devenir plus rentable, et, malgré de forts investissements, assure des revenus fabuleux (plus de 36 millions de florins entre 1733 et 1806) aux Thurn und Taxis, qui sont avec les Fugger les seuls anoblis de l'époque moderne à avoir réussi leur entrée dans la haute noblesse impériale. Au XVIIIe s., elle met même la main sur certaines de ses concurrentes territoriales, prenant par exemple à ferme en 1777 la poste autrichienne.

4 Mais l'essentiel, pour W.B., est en-dehors de la poste elle-même. Il réside dans la création d'un réseau dont le chemin de fer, voire l'internet, ne sont selon lui que les héritiers. La poste structure le temps : " la correspondance des amoureux, des 
diplomates et des marchands » (p. 126) adopte le rythme du jour de la poste, qui fait office d'arpentage du temps et de l'espace. « Une poste » devient en effet « une unité de mesure universelle » (p. 661) désignant à la fois une distance (environ 15 kilomètres), une durée (environ deux heures), un prix, et situe l'une envers l'autre les villes et les régions sur les cartes (l'une d'entre elles est reproduite en fin de volume), les itinéraires et les guides (un ars apodemica qui se codifie après le Raißbüchlein de l'Augsbourgeois Jörg Gail, 1563), les tarifs qui se multiplient et s'affinent progressivement, tandis que les impératifs du service unifient la mesure de l'heure. Le goût de la nouvelle lointaine, du savoir immédiat et toujours recommencé, se répand, dénoncé par les moralistes, repris par les auteurs de théâtre, multiplié enfin par la création des journaux. Le lien consubstantiel entre la poste et la presse est détaillé de manière très convaincante, des journaux à la main et recueils de nouvelles aux véritables périodiques, d'abord hebdomadaires - le premier naît à Strasbourg en 1605, et la poste sait si bien que ce nouveau type d'imprimé sourd d'elle-même qu'elle cherche à s'en assurer le monopole : c'est la Postzeitung de Francfort, créée par Birghden en 1617 et disparue en 1867 seulement. Multipolaire et doté d'une poste dynamique, le Saint-Empire est ainsi le berceau de la presse, il rassemble en 1669 encore $53 \%$ des lieux où s'impriment des journaux (p. 419). Le développement des coches impériaux suppose en outre des routes mieux entretenues : le réseau suscite son propre support et les exigences des maitres de poste déterminent l'amélioration du réseau routier impérial. Les petits territoires qui morcèlent l'Empire doivent s'entendre pour construire après 1712, à l'exemple français, des chaussées qui assurent à la fin du XVIIIe s. une supériorité routière à l'Ouest et au Sud de l'Empire (" ces régions de micro-principautés honnies par l'historiographie », p. 547) sur la Prusse, le Hanovre ou la Saxe.

5 Pour aller de Hambourg à Augsbourg, 30 jours sont nécessaires au début du XVIe s., seulement 5 à la fin. Entre temps, la perception du temps et de l'espace, de la nouveauté et du vaste monde, a changé - du moins pour ceux qui ont accès à la poste et utilisent ses coches, la géométrie et la diffusion sociales de l'innovation constituant le seul silence (même si le mot de « démocratisation » est hasardé p. 479) de ce livre peut-être trop prompt, dans un enthousiasme fervent envers sa propre réussite, à souligner la nouveauté de son propos (on a pourtant parlé de " première révolution des transports " avant même le recours incantatoire au terme de " communication ») et à relativiser l'importance des évolutions ultérieures. L'avènement de la poste est intronisé « mère de toutes les révolutions de la communication » (p. 42), mais les révolutions en ce domaine dépendent autant de la quantité des usagers que de l'inventivité du réseau et, indépendamment même du prix du billet, on assoit moins de passagers dans un coche que dans un train, de même que le courrier ou la presse de masse attendront la généralisation du timbre, de l'alphabétisation et du courrier administratif. Ne boudons cependant pas notre plaisir : ce livre, par l'abondance des connaissances et des dépouillements, par l'ampleur du propos, davantage encore par la capacité à intégrer des domaines trop souvent étanches (histoire du Saint-Empire, histoire des techniques, histoire sociale d'une institution, histoire des représentations), par la variété de ses méthodes (le chiffre n'est pas tabou - rareté outre-Rhin), par le brillant de la mise en œuvre et la fermeté des thèses, est une des grandes réussites de l'histoire moderne allemande lors de ces dernières années.

6 Christophe DUHAMELLE (MHFA) 